\title{
Distribuição longitudinal de sementes de soja com diferentes métodos de tratamento fitossanitário por um dosador de disco alveolar horizontal
}

\section{Dauto Pivetta CARPES ${ }^{1 *}$, Airton dos Santos ALONÇO ${ }^{1}$, Antônio Robson MOREIRA ${ }^{1}$, Gessieli POSSEBOM ${ }^{1}$, Arthur de Lima PIRES², Pablo do Amaral ALONÇO², Bruno Christiano Corrêa Ruiz ZART ${ }^{1}$}

\author{
${ }^{1}$ Programa de Pós-Graduação em Engenharia Agrícola, Universidade Federal de Santa Maria, Santa Maria, RS, Brasil. \\ ${ }^{2}$ Graduação em Agronomia, Universidade Federal de Santa Maria, Santa Maria, RS, Brasil. \\ *E-mail: dautocarpes@gmail.com
}

Recebido em outubro/2017; Aceito em dezembro/2017.

\begin{abstract}
RESUMO: A expressão do potencial genético da cultura da soja, possui relação direta com a qualidade da operação de semeadura, bem como, a utilização de sementes de elevado vigor e qualidade sanitária. $\mathrm{O}$ tratamento fitossanitário de sementes, é uma alternativa utilizada com a finalidade de proteger as sementes e plântulas nos estádios iniciais de desenvolvimento da cultura contra a ação de patógenos causadores de danos. Deste modo, objetivou-se avaliar diferentes métodos e produtos utilizados para o tratamento fitossanitário de sementes de soja, observando o seu efeito no desempenho de um dosador de disco alveolar horizontal. Os fatores observados foram os diferentes métodos de tratamento fitossanitário de sementes em quatro níveis, tratamento em tambor rotativo, adição de polímeros em tratamento industrial, tratamento industrial com adição de grafite e sementes sem adição de produto (testemunha). $\mathrm{O}$ segundo fator, foram as diferentes densidades de semeadura em quatro níveis (12, $14,16,18 \mathrm{sem} \mathrm{m}^{-1}$ ). As variáveis analisadas foram os espaçamentos entre sementes, aceitáveis, múltiplos, falhos e precisão. Após a análise e interpretação dos dados concluiu-se que, tanto o lubrificante sólido grafite como também os polímeros de recobrimento, podem ser utilizados sem que ocorra redução da qualidade de distribuição longitudinal de sementes com tratamento fitossanitário.
\end{abstract}

Palavras-chave: densidade de semeadura, dosador de precisão, polímeros adesivos.

\section{Longitudinal distribution of soybean seeds with different methods of phytosanitary treatment by a horizontal perforated disc meter}

\begin{abstract}
The expression of the genetic potential of the soybean crop is directly related to the quality of the sowing operation, as well as the use of high vigor seeds and sanitary quality. Phytosanitary treatment of seeds is an alternative used to protect seeds and seedlings in the early stages of development of the crop against the action of pathogens causing damage. In this way, the objective was to evaluate different methods and products used for the phytosanitary treatment of soybean seeds, observing its effect on the performance of a horizontal disk metering. The factors observed were the different methods of phytosanitary treatment of seeds at four levels, rotary drum treatment, addition of polymers in industrial treatment, industrial treatment with addition of graphite and seeds without addition of product (control). The second factor was the different sowing densities at four levels $\left(12,14,16,18\right.$ without $\left.\mathrm{m}^{-1}\right)$. The analyzed variables were seed spacings, acceptable, multiple, fail and precision. After the analysis and interpretation of the data, it was concluded that both the graphite solid lubricant and the coating polymers can be used without reducing the quality of the longitudinal distribution of seeds with phytosanitary treatment.
\end{abstract}

Keywords: seeding density, metering precision, adhesive polymers.

\section{INTRODUÇÃO}

Um dos principais fatores que pode determinar a produtividade de uma cultura é a qualidade da operação de semeadura bem como a utilização de sementes de elevada qualidade genética e sanitária. Krzyzanowski et al. (2008) afirma que sementes de soja de elevado potencial fisiológico podem proporcionar incremento no rendimento de grãos superiores a $35 \%$ em comparação a sementes de baixo potencial. Porém, além do elevado potencial da semente, é necessário observar uma série de fatores que envolvem o ambiente de germinação e desenvolvimento das plântulas.

De acordo com Baudet; Peske (2006) o tratamento fitossanitário de sementes, pode garantir índice adequado de emergência das plântulas, bem como seu desenvolvimento protegido contra a ação de insetos e fungos nos estádios iniciais do ciclo vegetativo. Além de tratamentos fitossanitários, de acordo com Nascimento et al. (1993) o recobrimento de sementes com materiais inertes é uma técnica que possibilita a ação simultânea de nutrientes, fungicidas, inseticidas, herbicidas e microorganismos benéficos a cultura.

Com relação ao desempenho das máquinas que fazem a semeadura das culturas, Carpes et al. (2013), afirmam que os produtos utilizados para tratamento fitossanitário ocasionam a alteração da rugosidade da semente, conferindo uma certa aderência entre as sementes e consequentemente, reduzindo o escoamento das mesmas no reservatório do mecanismo dosador, o que ocasiona a dificuldade de captação e individualização principalmente por mecanismos dosadores de disco horizontal. 


\section{Carpes et al.}

A desuniformidade da distribuição longitudinal de sementes poderá favorecer a ocorrência de espaçamentos duplos ocasionando o acúmulo de plantas em determinados pontos da linha de semeadura, gerando plantas com maior altura, reduzida ramificação e menor diâmetro de caule, tornando-as susceptíveis ao acamamento. Havendo falhas na distribuição de sementes, é favorecido o surgimento de plantas de soja com tamanho reduzido, além de favorecer o surgimento de plantas daninhas pela ineficiência de fechamento do dossel da cultura influenciando a redução de produtividade (PINHEIRO NETO et al., 2008).

Segundo Mantovani et al. (1999) e Hentschke (2002) para minimizar esse efeito, é recomendado que seja realizada a adição de lubrificante sólido grafite, material inerte que reduz o atrito entre as sementes facilitando o escoamento e adequação aos alvéolos do disco dosador.

Outro método adotado para garantir um correto desempenho de mecanismos dosadores é a utilização de polímeros adesivos, de acordo com Kunkur et al. (2007) esses produtos formam uma película de recobrimento sobre a semente, melhorando a uniformidade de espalhamento e ação de fungicidas, inseticidas e micronutrientes, além de proporcionar maior facilidade de escoamento, podendo inclusive dispensar o uso de lubrificante sólido grafite, contribuindo para que a mesma possa ser depositada no solo em local adequado e, dessa forma, expressar todo seu potencial genético em termos fisiológicos desde a fase inicial de desenvolvimento.

O objetivo deste trabalho foi avaliar diferentes métodos e produtos utilizados para o tratamento fitossanitário de sementes de soja, observando o seu efeito no desempenho de um dosador de disco alveolar horizontal, através da verificação da distribuição longitudinal de sementes de soja com ensaios em laboratório.

\section{MATERIAL E MÉTODOS}

O experimento foi conduzido nas instalações do Laboratório de Pesquisa e Desenvolvimento de Máquinas Agrícolas (LASERG), vinculado à Universidade Federal de Santa Maria.

Foi utilizada uma bancada de ensaio de dosadores desenvolvida por Silveira et al. (2010) que permite o acoplamento e acionamento de diversos modelos de mecanismos dosadores de sementes. A movimentação dos discos é realizada por um motor elétrico de indução assíncrono, com potência de 736 Watt (W) e 1720 rotações por minuto (rpm), controlada com auxílio de um inversor de frequência CFW - 08 com entrada monofásica de 200 a 240 Volts (V).

A transmissão do movimento do motor para o dosador é feita através de uma correia ligada a um par de polias (motora e movida), a redução da rotação é feita através de um redutor de engrenagem com relação de transmissão de 1:11 reduzindo 19,64 vezes a rotação do motor. No decorrer dos ensaios, a bancada com os dosadores permaneceu nivelada longitudinalmente e transversalmente, e o reservatório de sementes dos dosadores foi mantido no nível cheio.

Também foi utilizada uma esteira revestida com feltro, medindo 17,5 metros (m) de comprimento e $0,15 \mathrm{~m}$ de largura onde são depositadas as sementes dosadas. Ao movimentar-se sob o mecanismo dosador de sementes, ocorre a simulação da velocidade de avanço horizontal da semeadora no solo. Seu acionamento é realizado por um motor elétrico trifásico de indução assíncrono de $2208 \mathrm{~W}$, acionado por um inversor de frequência CFW - 10, com entrada monofásica de 200 a 240 $\mathrm{V}$, descrito por (SILVEIRA et al., 2010). A velocidade de deslocamento simulada para as avaliações foi de $1,67 \mathrm{~m} \mathrm{~s}^{-1}$, intermediária às velocidades recomendadas pela norma ISO $7256 / 1$, a qual cita as velocidades de 1,39 e $1,94 \mathrm{~m} \mathrm{~s}^{-1}$.

Na Tabela 1 são apresentadas as configurações utilizadas no mecanismo dosador de sementes de disco alveolar horizontal STARA para proporcionar as densidades de semeadura pré-estabelecidas com espaçamento entre linhas de $0,45 \mathrm{~m}$ e respectivos espaçamento de referência entre sementes $\left(\mathrm{X}_{\mathrm{ref}}\right)$.

Tabela 1. Descrição da densidade de semeadura $\left(\operatorname{Sem~} \mathrm{m}^{-1}\right)$, espaçamento de referência entre sementes (Xref), rotação do disco dosador (RD) e velocidade angular do disco dosador (VA).

Table 1. Description of seeding density $\left(\right.$ seeds $\mathrm{m}^{-1}$ ), reference seed spacing $\left(\mathrm{X}_{\mathrm{ref}}\right)$, metering disk rotation $(\mathrm{RD})$ and angular velocity of the feeder disk (VA).

\begin{tabular}{ccccc}
\hline Sem ha $^{-1}$ & Sem m $^{-1}$ & $\mathrm{X}_{\text {ref }}(\mathrm{m})$ & $\mathrm{RD}(\mathrm{rpm})$ & VA $\left(\mathrm{rad} \mathrm{s}^{-1}\right)$ \\
\hline 250.000 & 12 & 0,08 & 14,06 & 1,47 \\
300.000 & 14 & 0,07 & 16,87 & 1,77 \\
350.000 & 16 & 0,06 & 19,68 & 2,06 \\
400.000 & 18 & 0,05 & 22,5 & 2,36 \\
\hline \multicolumn{2}{l}{ Sem ha ${ }^{-1}-$ Sementes por hectare; Sem m } \\
-1
\end{tabular}

V.A. - Velocidade Angular do Disco Dosador; RD - Rotação do disco dosador

Foram utilizadas sementes de soja (Glycine max L.) Nidera ${ }^{\circledR}$ 5909, peneira 8. As densidades de semeadura estabelecidas estão de acordo com as recomendações técnicas para a cultivar utilizada.

O disco alveolar horizontal do mecanismo dosador foi selecionado admitindo-se uma folga entre o alvéolo e a semente de aproximadamente $10 \%$ maior que a máxima dimensão das mesmas, de modo que não proporcione uma dúplice dosagem e nem a obstrução das sementes. De acordo com Kepner et al. (1982) essa recomendação contribui para um correto preenchimento e exclusão das sementes dos alvéolos do disco dosador.

A descrição dos métodos e formas de tratamentos aplicados nas sementes utilizadas estão apresentados na Tabela 2. Foram utilizadas sementes com ausência de produtos fitossanitários constituindo o tratamento testemunha (SP), sementes com tratamento (Fungicida + Inseticida) realizados em tambor rotativo de eixo excêntrico (STR), sementes com tratamento mais recobrimento com polímero realizados com aplicação industrial (STI) e sementes tratadas industrialmente com adição de lubrificante sólido grafite (STG).

A aferição dos espaçamentos foi realizada com uso de uma fita métrica estendida paralelamente sobre a esteira, os dados foram digitados em planilhas eletrônicas onde posteriormente foram classificados conforme norma (ISO 7256/1, 1982), a qual classifica os espaçamentos em aceitáveis (aqueles entre 0,5 e 1,5 vezes o espaçamento médio de referência $X_{\text {ref.), }}$, múltiplos (menores que 0,5 vezes o $\mathrm{X}_{\text {ref. }}$ ) e falhos (maiores que 1,5 vezes o $\left.X_{\text {ref. }}\right)$.

Outra variável observada foi a precisão do mecanismo para a dosagem de sementes, a mesma mensura a equidistância dos espaçamentos entre sementes e é determinada pela equação 1, onde DP é o Desvio Padrão dos espaçamentos e $\mathrm{X}_{\text {ref }}$ é o espaçamento teórico nominal entre sementes. 
Precisão $=($ DP $/$ Xref $) * 100$

(Equação 1)

Tabela 2. Descrição dos tratamentos fitossanitários utilizados nas sementes de soja

Table 2. Description of the phytosanitary treatments used in soybean seeds.

\begin{tabular}{|c|c|}
\hline Semente & Descrição do tratamento \\
\hline (STR) & $\begin{array}{l}2 \text { mililitro }(\mathrm{mL}) \text { de Standak Top por quilograma }(\mathrm{kg}) \mathrm{de} \\
\text { sementes }\end{array}$ \\
\hline (STI) & $\begin{array}{l}4 \mathrm{~mL} \mathrm{~kg}^{-1} \text { Cropstar }+2 \mathrm{~mL} \mathrm{~kg}^{-1} \text { Derosal Plus }+2 \mathrm{~mL} \mathrm{~kg}^{-} \\
{ }^{1} \text { Peridian Blue }+3 \mathrm{~g} \mathrm{~kg}^{-1} \text { TalkunGlos }+2 \mathrm{~mL} \mathrm{~kg} \\
\text { FertiactylLeg } 02\end{array}$ \\
\hline (STG) & $\begin{array}{l}\text { Derosal } 2 \mathrm{~mL} \mathrm{~kg}^{-1} \text { de sementes }+4,0 \text { gramas }(\mathrm{g}) \mathrm{de} \\
\text { grafite por kg de semente }\end{array}$ \\
\hline
\end{tabular}

O número de espaçamentos observados foi de acordo com o estabelecido pelo projeto de norma de avaliação de semeadoras de precisão da ABNT, (1994), sendo estabelecido para cultura da soja, a coleta de 250 espaçamentos entre sementes.

Foi utilizado o delineamento inteiramente casualizado com quatro repetições em arranjo fatorial $4 \times 4$, totalizando sessenta e quatro unidades experimentais. Os tratamentos foram compostos por quatro densidades de semeadura: $12,14,16,18$ sementes por metro linear $\left(\mathrm{sem} \mathrm{m}^{-1}\right)$, e três métodos de tratamento fitossanitário mais a testemunha; sementes tratadas em tambor rotativo (STR), sementes com polímero tratadas industrialmente, sementes tratadas industrialmente com adição de lubrificante sólido grafite e sementes sem produto (testemunha).

Os dados foram submetidos previamente aos testes de Kolmorogov-Smirnov e Cochran, afim de verificar a conformidade dos resíduos a distribuição normal e homogeneidade das variâncias, respectivamente. Constatada a condição de normalidade e homogeneidade, procedeu-se a análise de variância (ANOVA, $\mathrm{P}<0,05)$ no software ASSISTAT versão 7.7 (2016). Médias dos tratamentos qualitativos foram comparadas pelo teste de Tukey $(\mathrm{P}<0,05)$, enquanto os quantitativos submetidos a análise de regressão $(\mathrm{P}<0,05)$.

\section{RESULTADOS}

Os resultados da análise de variância estão expostos na Tabela 3, não houve interação significativa do método de tratamento fitossanitário com a densidade de semeadura sobre as variáveis, espaçamentos aceitáveis, múltiplos, falhos e precisão a $1 \%$ e $5 \%$ de probabilidade de erro respectivamente. Os percentuais de espaçamentos aceitáveis, múltiplos, falhos e precisão estão expostos na Tabela 4.

$\mathrm{Na}$ Figura 1, são expostas as variações dos percentuais de espaçamentos entre sementes para cada densidade de semeadura avaliada.

\section{DISCUSSÃO}

$\mathrm{O}$ coeficiente de variação $(\mathrm{CV})$ para todas as variáveis avaliadas manteve-se dentro do limite proposto por Coelho (1996), o autor recomenda um CV de no máximo 50\% para avaliação de dosadores mecânicos de disco alveolar horizontal.

Não houve interação entre os fatores métodos de tratamento e densidade de semeadura, porém, os maiores percentuais de espaçamentos aceitáveis, foram obtidos para as sementes com tratamento industrial e recobertas por polímero adesivo, demonstrando também os menores índices de espaçamentos múltiplos e falhos, porém, não diferiram estatisticamente das sementes que possuem tratamento industrial mais adição de lubrificante sólido grafite, conforme os dados apresentados na Tabela 4.

Os resultados obtidos estão abaixo dos valores encontrados por Jasper et al. (2006), os autores avaliaram a distribuição longitudinal de sementes de milho com diferentes formas de mistura e tratamento fitossanitário, obtiveram média de 96,88\% de espaçamentos aceitáveis. O percentual médio de precisão dos espaçamentos não ultrapassou o limite de $29 \%$ estabelecido por Kachman; Smith (1995), dessa forma, ocorreu uma boa regularidade dos espaçamentos entre sementes.

A utilização de polímero de recobrimento nas sementes pode proporcionar o mesmo efeito da utilização do lubrificante sólido grafite, fazendo com que as mesmas tenham uma boa escoabilidade dentro do reservatório de sementes facilitando a captação pelos alvéolos do disco dosador. O recobrimento com polímero além de contribuir com o melhor espalhamento superficial dos produtos fitossanitários, também proporciona o aumento de uniformidade dimensional das sementes corroborando as informações de Nascimento (2000), esta técnica pode melhorar a plantabilidade das sementes, colaborando para o correto preenchimento dos alvéolos, permitindo o alojamento individual das sementes e reduzindo o percentual de captação múltipla.

A Figura 1A demonstra a variação das porcentagens de espaçamentos para o tratamento testemunha, onde houve uma maior ocorrência de falhos. Este fator pode ter origem devido a quantidade de espaçamentos múltiplos originados, levandose em consideração que um espaçamento múltiplo é observado quando as sementes estão mais próximas umas das outras isto acarreta em um maior espaço para a semente em sequência, originando um espaço falho na linha de semeadura.

Tabela 3. Resultados da análise de variância para as variáveis espaçamentos aceitáveis, múltiplos, falhos e precisão.

Table 3. Results of variance analysis for the variables acceptable, multiple, fail and precision spacing.

\begin{tabular}{lcccc}
\hline & Aceitáveis & Múltiplos & Falhos & Precisão \\
\hline Fator de variação & $\mathrm{p}$ & $\mathrm{p}$ & $\mathrm{p}$ & $\mathrm{p}$ \\
Tratamento (F1) & 0.0001 & 0.0012 & 0.0001 & 0.092 \\
Densidade (F2) & -- & -- & -- & -- \\
F1 x F2 & 0.2493 & 0.1357 & 0.4672 & 0.0001 \\
MG & 61.52 & 19.81 & 18.65 & 26.69 \\
CV $(\%)$ & 8.34 & 15.16 & 15.69 & 4.86 \\
\hline
\end{tabular}

** significativo ao nível de $1 \%$ de probabilidade $(\mathrm{p}<.01) ; *$ significativo ao nível de $5 \%$ de probabilidade $(.01=<\mathrm{p}<.05)$; ns não significativo $(\mathrm{p}>=.05)$; $\mathrm{p}=\mathrm{p}$ Valor; F2 - Quantitativo, teste f não se aplica

Tabela 4. Resultado do desdobramento de médias para distribuição longitudinal entre sementes de soja e precisão do dosador

Table 4. Results of the unfolding of averages for longitudinal distribution soybean seeds and metering accuracy.

\begin{tabular}{lcccc}
\hline \multirow{2}{*}{ Tratamento } & \multicolumn{4}{c}{ (\%) Espaçamentos entre sementes } \\
\cline { 2 - 5 } & Aceitáveis & Múltiplos & Falhos & Precisão \\
\hline (SP) & $55.48 \mathrm{c}$ & $20.19 \mathrm{ab}$ & $24.31 \mathrm{a}$ & $27.42 \mathrm{a}$ \\
(STR) & $59.65 \mathrm{bc}$ & $22.15 \mathrm{a}$ & $18.18 \mathrm{~b}$ & $26.48 \mathrm{a}$ \\
(STI) & $66.88 \mathrm{a}$ & $17.69 \mathrm{~b}$ & $15.41 \mathrm{c}$ & $26.37 \mathrm{a}$ \\
(STG) & $64.06 \mathrm{ab}$ & $19.21 \mathrm{~b}$ & $16.71 \mathrm{bc}$ & $26.51 \mathrm{a}$ \\
\hline DMS & 4.82 & 2.82 & 2.75 & 1.22 \\
\hline
\end{tabular}

MG Média geral; CV (\%) Coeficiente de variação; DMS Diferença mínima Significativa; *Médias não seguidas de mesma letra na coluna, diferem pelo teste de Tukey em nível de 5\% de probabilidade de erro. 
(A)

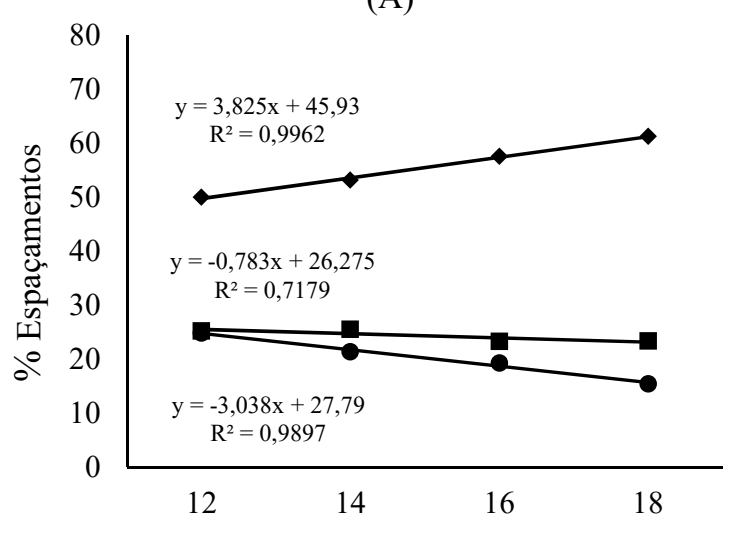

(C)

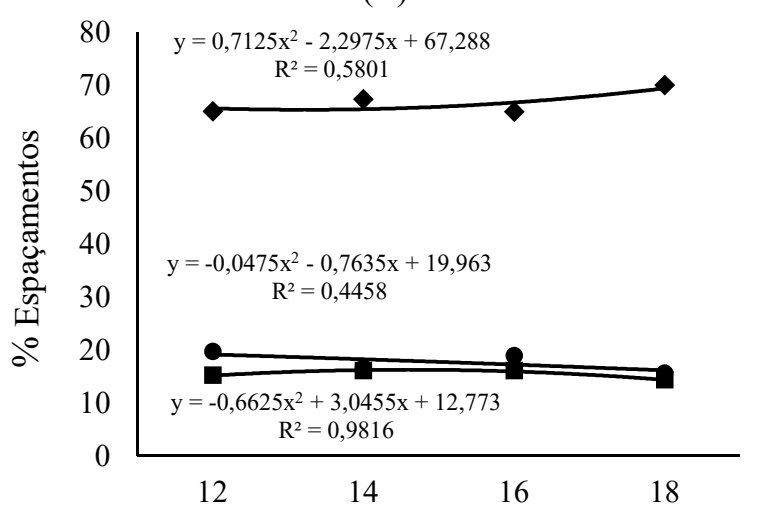

(B)

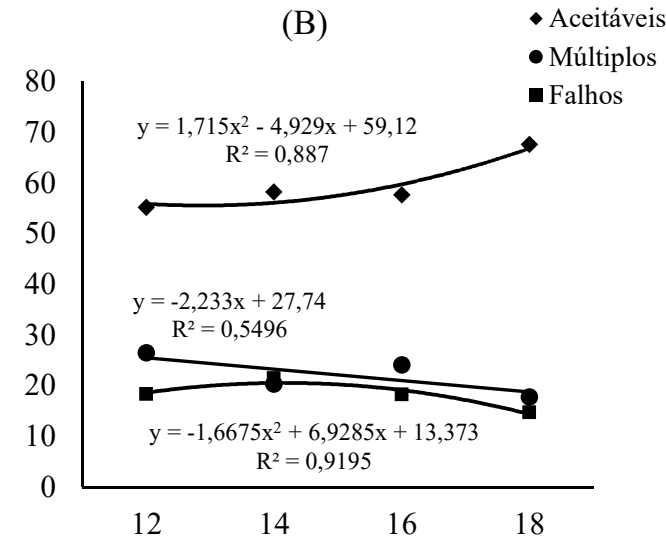

(D)

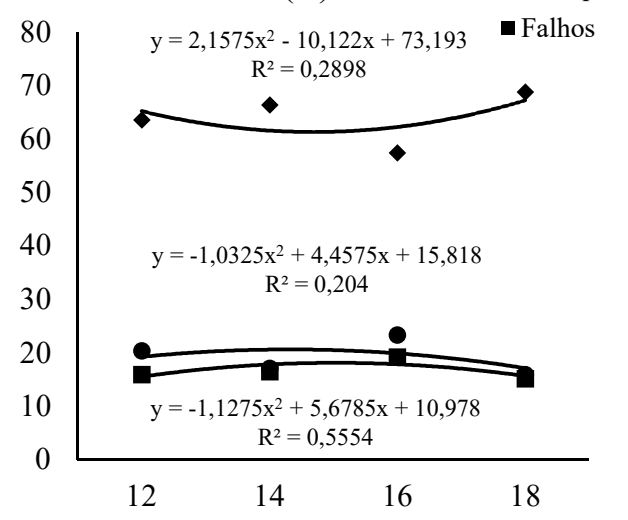

Densidade de Semeadura $\left(\mathrm{sem} \mathrm{m}^{-1}\right)$

Figura 1. Percentuais de espaçamentos aceitáveis, múltiplos e falhos conforme elevação da densidade de semeadura para, (A) Tratamento Testemunha (SP); (B) Tratamento Tambor Rotativo (STR); (C) Tratamento Industrial + Polímero (STI); (D) Tratamento + Grafite (STG). Figure 1. Percentages acceptable spacing, as multiple and fail elevated density for seeding (A) Treatment Control (SP); (B) Treatment Rotating Drum (STR); (C) Industrial + Polymer Treatment (STI); (D) Treatment + Graffiti (STG).

Para os tratamentos STR, STI e STG Figuras B, C e D, respectivamente, foi observado um comportamento não linear dos espaçamentos aceitáveis conforme foi elevado o número de sementes por metro, corroborando os resultados encontrados por Reis et al. (2007) que não obtiveram resposta linear da regularidade de distribuição de sementes com aumento da velocidade, os autores obtiveram o melhor percentual de espaçamentos aceitáveis em uma velocidade intermediária entre as três estudadas, porém, discordam das conclusões encontradas por Dias (2014) que obteve resposta linear para a redução dos espaçamentos aceitáveis ao elevar a velocidade periférica dos discos dosadores.

Para a menor densidade avaliada não houve desempenho satisfatório, sendo a maior porcentagem alcançada para o STR, com $65,10 \%$ de espaçamentos aceitáveis. Estes resultados contestam as conclusões de Bozdoğan (2008) o autor afirma que, quanto menor a velocidade periférica do disco dosador, melhor o desempenho. O provável fator para este acontecimento pode ser explicado devido ao fato de o dosador possuir uma faixa de velocidade periférica mais adequada para uma determinada densidade de semeadura.

Foram observados distintos comportamentos para as variáveis verificadas em função dos diferentes métodos de tratamento. Nas Figuras B, C e D foi observado que o mecanismo dosador, na maior densidade, proporcionou porcentagens de espaçamentos aceitáveis próximas a $70 \%$, estando dentro da faixa de desempenho recomendada por (COELHO, 1996). O autor cita que dosadores de discos horizontais, devem apresentar desempenho em torno de $60 \%$ ou mais de espaçamentos aceitáveis.

\section{CONCLUSÕES}

Os resultados demonstraram que tanto o lubrificante sólido grafite como também os polímeros de recobrimento, são alternativas que podem ser utilizadas sem que haja redução significativa da qualidade de distribuição longitudinal e, consequentemente, um adequado nível de precisão na equidistância dos espaçamentos entre as sementes.

\section{REFERÊNCIAS}

ABNT. Associação Brasileira de Normas Técnicas. Projeto de Norma 04:015:06-004. Semeadora de precisão: ensaio de laboratório. São Paulo, 1994. 22p.

BAUDET, L.; PESKE, T.S. A logística do tratamento de sementes. Revista Seed News, Pelotas, v. 10, p. 22-25. 2006. Disponível

em: 
$<$ http://www.seednews.inf.br/portugues/seed101/artigocapa 101.shtml> Acesso em: 15 jul 2017.

BOZDOĠAN, A. M. Seeding uniformity for vacuum precision seeders. Scientia Agrícola, Piracicaba, v. 65, n. 3, p. 318322, 2008. DOI: http://dx.doi.org/10.1590/S010390162008000300013

CARPES, D. P.; ALONÇO, A. dos S.; FRANCETTO, T. R.; BELLÉ, M. P.; VEIT, A. A. Uso obrigatório. Revista Cultivar Máquinas, v. 130, p. 6-8, 2013.

COELHO, J.L.D. Ensaio \& certificação das máquinas para a semeadura. In MIALHE, L.G. Máquinas agrícolas: ensaio \& certificação. Piracicaba: Fundação de Estudos Agrários Luiz de Queiroz, 1996. p. 551-569.

DIAS, V. de O.; ALONÇO, A. dos S.; CARPES, D. P.; VEIT, A. A.; SOUZA, L. B. Tamanho amostral para ensaios de mecanismos dosadores de sementes de milho em esteira carpetada. Engenharia Agrícola, Jaboticabal, v. 34, p. 1019-1029, 2014. http://dx.doi.org/10.1590/S010069162014000500021.

HENTSCHKE, C. Cultura do milho: planejamento do plantio. Revista Seed News, Pelotas, n. 4, p. 18-20, 2002. Disponível em: $<$ http://www.seednews.inf.br/portugues/seed64/milho64.sh tml> Acesso em: 06 ago 2017.

International Organization for Standardization. ISO: 7256/1: Sowing equipment - methods of test: part 1 . Single seed drills (Precision drills). Geneva, 16 p., 1982.

JASPER, R.; JANSZEN, U.; JASPER, M.; GARCIA, L. C.; Distribuição longitudinal e germinação de sementes de milho com emprego de tratamento fitossanitário e grafite. Engenharia Agrícola, Jaboticabal, v. 26, n. 1, p. 292-299, 2006. DOI: http://dx.doi.org/10.1590/S010069162006000100031

MANTOVANI, E.C.; MANTOVANI, B.H.M.; CRUZ, I.; MEWES, W.L.C.; OLIVEIRA, A.C. Desempenho de dois sistemas distribuidores de sementes utilizados em semeadoras de milho. Pesquisa Agropecuária Brasileira, Brasília, v. 34, n. 1, p. 93-8, 1999. DOI: http://dx.doi.org/10.1590/S0100-204X1999000100013.

NASCIMENTO, W. M. Hortaliças: Tratamento de sementes. Revista Seed News. Pelotas, v. 4, n. 2, p. 16-17, 2000. Disponível em: $<$ http://www.seednews.inf.br/_html/site/content/reportage m_capa/index.php?edicao $=11 \overline{6}>$ Acesso em: 02 jun 2017 .

NASCIMENTO, W.; SILVA, J.; MARTON, L. Qualidade fisiológica de sementes peletizadas de tomate durante o armazenamento. Informativo ABRATES, Londrina, v. 3, n. 3, p. 47, 1993. Disponível em: $<$ http://www.scielo.br/pdf/rbs/v25n2/19645.pdf $>$ Acesso em: 15 jul 2017.
KACHMAN, S. D.; SMITH, J. A. Alternative measures of accuracy in plant spacing for planter susing single seed metering. Transactions of the ASAE, St Joseph, v. 38, n. 2, p. 379-387, 1995 . DOI: https://dx.doi.org/10.13031/2013.27843)

KEPNER, R. A.; BAINER, R.; BARGER, E. L. Principles of farm machinery. Westport, Connecticut: Avi Publishing Company. Crop Planting 10, p. 209-35, 1982.

KRZYZANOWSKI, F. C.; FRANÇA NETO, J. de B.; HENNING, A. A.; COSTA, N. P. Semente de Soja como Tecnologia e Base para Altas Produtividades. Circular Técnica 55. Série Sementes. EMBRAPA-CNPSO. Londrina, Brasil. 2008. 8p. Disponível em: < https://www.embrapa.br/soja/busca-de-publicacoes//publicacao/457138/a-semente-de-soja-como-tecnologia-ebase-para-altas-produtividades-serie-sementes $>$ Acesso em: 15 jul 2017.

KUNKUR, V.; HUNJE, R.; PATIL, N.K.B.; VYAKARNHAL, B.S. Effec to seed coating with polymer, fungicide and insecticide on seed quality in cotton during storage. Karnataka Journal of Agricultural Sciences, v. 20, n. 1, p. 137-139, 2007. Disponível em: $<$ http://203.129.218.157/ojs/index.php/kjas/article/view/42/ 42> Acesso em: 02 jun 2017.

PINHEIRO NETO, R.; BRACCINI, A. L.; SCAPIM, C. A.; BORTOLOTTO, V. C.; PINHEIRO, A. C. Desempenho de mecanismos dosadores de sementes em diferentes velocidades e condições de cobertura de solo. Acta Scientiarum Agronomy, Maringá, v. 30, p. 611-617, 2008. REIS, E. F. Características operacionais de uma semeadoraadubadora de plantio direto na cultura da soja (Glycine Max (L.) Merril). Revista Ciências Técnicas Agropecuárias, Havana, v. 16, n. 3, p. 70-75, 2007. Disponível em: $<$ http://www.redalyc.org/pdf/932/93216315.pdf> Acesso em: 02 jun 2017.

SILVA, F. de A. S.; AZEVEDO, C. A. V. de. The Assistat Software Version 7.7 and its use in the analysis of experimental data. African Journal of Agricultural Research, v. 11, n. 39, p. 3733-3740, 2016. DOI: https://dx.doi.org/10.5897/AJAR2016.11522

SILVEIRA, H. A. T. da.; ZOTTIS, J.; DIAS, V. de O.; BAUNHARDT, U. B.; ALONÇO, A dos S. Projeto e desenvolvimento de uma bancada de ensaios de dosadores pneumáticos: fase preliminar e detalhada. In: Congresso Brasileiro de Engenharia Agrícola. 39, 2010, Vitória, Anais... Jaboticabal: SBEA, 4p. 